\title{
Gene expression in bovine mammary somatic cells isolated from milk*
}

\author{
A.A. Hayashi ${ }^{1}$, S.A. McCoard ${ }^{1,3}$, N.C. Roy ${ }^{1}$, M.P.G. Barnett ${ }^{1}$, \\ D. D.S. Mackenzie ${ }^{2}$ and W.C. McNabb ${ }^{1}$ \\ ${ }^{1}$ Nutrition and Behaviour Group, AgResearch Limited \\ ${ }^{2}$ Institute of Food, Nutrition and Human Health, Massey University \\ Palmerston North, New Zealand
}

\begin{abstract}
Traditionally, in order to examine the expression of genes involved in mammary gland metabolism, RNA was prepared from mammary biopsies or from tissue samples taken at slaughter. The objective of this study was to validate the use of milk somatic cells as a model to study gene expression in the lactating bovine mammary gland. The expression pattern of milk protein and epithelial cell marker genes was similar between somatic cells and mammary tissue indicating that cells exfoliated into milk provide a viable non-invasive tissue source for gene profiling in the bovine mammary gland.
\end{abstract}

KEY WORDS: mammary gland, gene profiling, protein synthesis, somatic cells

\section{INTRODUCTION}

The physiological status of a cell or organism is, to a large extent, directed by the complement of genes expressed. Gene profiling studies usually require tissues collected using invasive techniques such as biopsies or tissue samples taken at slaughter. Somatic cells present in milk provide a potential alternative non-invasive source of mammary tissue. They are present in the milk throughout lactation and consist primarily of leukocytes and epithelial cells exfoliated from the mammary epithelium. Boutinard et al. (2002) reported that somatic cells

\footnotetext{
${ }^{*}$ Supported by AgResearch Strategic Investment, the Foundation for Research, Science and Technology, a FRST Postdoctoral Fellowship (Sue McCoard) and a CAPES Brazilian Doctoral Fellowship (Amanda Hayashi) and is part of a larger mammary genomics Joint Venture in collaboration with Primary Industry Research Victoria, Australia

${ }^{3}$ Corresponding author: e-mail: sue.mccoard@agresearch.co.nz
} 
exfoliated from the mammary epithelium during the milking process provided an alternative non-invasive source of mammary tissue for gene expression profiling in goats. This approach potentially offers significant improvement over the use of biopsy and post-mortem sampling for assessing gene expression. Additionally, the use of milk somatic cells could allow repeated gene expression profiling during the lactation period using the same animal. To date, this approach for bovine mammary gene expression studies has not been evaluated.

Therefore, our hypothesis was that somatic cells isolated from milk would provide a useful alternative tissue source for gene expression profiling in the bovine mammary gland. To validate the use of milk somatic cell RNA for gene expression profiling in the bovine mammary gland, we compared the profiles of the major milk protein genes and selected epithelial cell markers between mammary tissue and milk somatic cells collected from cows treated with growth hormone $(\mathrm{GH})$ and untreated control animals.

\section{MATERIAL AND METHODS}

Milk somatic cells were collected from 8 spring-calved, first lactation (midlate) Jersey cows, randomly allocated to control (saline) and treatment (single subcutaneous injection of commercial GH; Lactatropin ${ }^{\circledR}$, Elanco Animal Health). All cows were maintained under identical environment and management practices, fed a nutritionally balanced total mixed ration ad libitum and milked twice daily. After six days of treatment, all cows were milked and immediately euthanised. Total cellular RNA was isolated from milk somatic cells using Trizol (Invitrogen) and purified using RNeasy columns (Qiagen). Only three cows per treatment group yielded sufficient quantity of RNA from somatic cells to conduct the experiment. Total RNA was converted to fluorescently-labelled cDNA (SuperScript cDNA Labelling System, Invitrogen) and hybridized to 23.000 bovine expressed sequence tag (EST) microarrays printed on glass slides. The slides were scanned using GenePix 5.0 software. Microarray data were normalized and analysed using REML (Baird et al., 2002, 2003) and a list of genes differentially expressed in response to GH treatment were compared with expression profiles from post-mortem tissues from the same cows (McCoard et al., 2004, companion paper).

The ESTs were selected from AgResearch's proprietary database that represents approximately $80 \%$ of the bovine genome. The arrays used for the comparison of mammary tissue and somatic cells contained different sets of ESTs. However, both arrays contained ESTs representing the milk protein genes and epithelial cell markers described in this study. 


\section{RESULTS}

Comparison of gene expression profiles showed that the expression of the genes encoding $\kappa$-casein, $\beta$-casein, $\alpha_{\mathrm{s} 1}$-casein, $\alpha$-lactalbumin were similar between somatic cells and post-mortem mammary tissue collected from the same animal (Table 1). However, $\beta$-lactoglobulin gene expression was down-regulated in somatic cells and up-regulated in mammary tissue. The pattern of expression of the genes encoding selected epithelial cell markers was similar between somatic cells and mammary tissue (Table 1).

Table 1. Effect of GH on milk protein and epithelial cell marker genes expression relative to controls $(\mathrm{P}<0.05)$ in simultaneously collected post-mortem mammary tissue and milk somatic cells

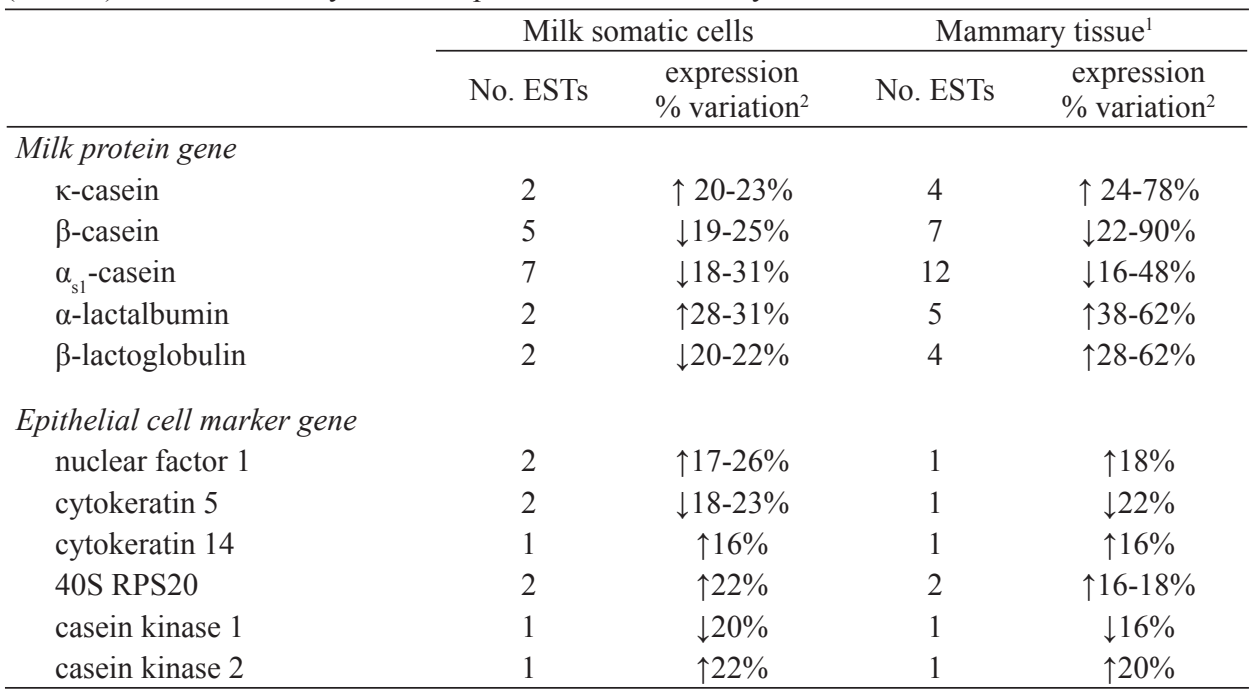

${ }^{1}$ data derived from McCoard et al., 2004 (companion paper)

$2 \uparrow$ - up-regulated expression; $\downarrow$ - down-regulated expression

\section{DISCUSSION}

This study demonstrates the functional use of RNA extracted from milk somatic cells for assessing gene expression profiles in the bovine mammary gland. It has been reported that somatic cells accurately reflect events occurring in the goat mammary gland (Boutinard et al., 2002) but this is the first study to validate the use of milk somatic cells for gene expression studies in the cow. Comparison of the expression profile of milk protein genes between milk somatic cells and post-mortem tissues showed the same pattern in the two RNA samples. Miyoshi et al. (2002) and Tammen et al. (2003) reported that cytokeratin 5, cytokeratin 14 and nuclear factor-1 are good markers for mammary epithelial cells and can 
be used as markers for differentiation of epithelial cells during carcinogenesis in humans. Our study confirms that the pattern of gene expression for these and others epithelial cell markers and milk protein genes are similar between mammary tissue and milk somatic cells.

\section{CONCLUSIONS}

This study indicates that milk somatic cells accurately reflect gene expression profiles in lactating mammary tissue. This method is a significant improvement over the use of biopsy samples and allows easy and repetitive sampling without damage to the mammary gland. Thus, gene expression profiling, as determined via RNA from somatic cells may provide a convenient means to more dynamically characterize events within the bovine mammary gland.

\section{REFERENCES}

Baird D., Johnstone P., Wilson T., 2002. The analysis of micro array data using mixed models. Workshop on the Design and Analysis of Micro array/Chip Experiments. June 2002, Melbourne (Australia)

Baird D., Johnstone P., Wilson T., 2003. Normalization of micro array data using a spatial mixed model in a REML analysis. Bioinformatics (accepted)

Boutinard M., Rulquin H., Keisler D.H., Djiane J., Jammes H., 2002. Use of somatic cells from goat milk for dynamic studies of gene expression in the mammary gland. J. Anim. Sci. 80, 12581269

Miyoshi K., Meyer B., Gruss P., Cui Y., Renou J., Morgan F.V., Smith G.H., Reischenstein M., Shani M., Hennighausen L., Robinson G.W., 2002. Mammary epithelial cells are not able to undergo pregnancy-depedent differentiation in the absence of the helix-loop-helix inhibitor Id2. Mol. Endocrinol. Soc. 16, 2892-2901

Tammen H., Kreipe H., Hess R., Kellmann M., Lehmann U., Pich A., Lamping N., Knappe P., Zucht H., Lilischkis R., 2003. Expression profiling of breast cancer cells by differential peptide display. Breast Cancer Res. 79, 83-93 